\title{
Solubilization of Oleic Acid by Myrj 59 Surfactant
}

\author{
AMIRA SATIRAWATY BT MOHD PAUZAN ${ }^{1 *} \&$ MADZLAN AZIZ ${ }^{2}$ \\ ${ }^{1}$ Department of Chemistry, Faculty of Resource Science and Technology, Universiti Malaysia Sarawak, \\ 94300 Kota Samarahan, Sarawak, Malaysia; ${ }^{2}$ Department of Chemistry, Faculty of Science, Universiti \\ Teknologi Malaysia, 81310 Skudai, Johor, Malaysia
}

\begin{abstract}
Palm oil mill effluent (POME) is one of the sources of contamination in effluent that leads to problems such as clogging in drainpipes and sewer lines. POME discharge consists of high content of free fatty acids (FFAs) as well as high concentration of biochemical oxygen demand (BOD), chemical oxygen demand and suspended solids. FFAs in effluent are not favorable due to low water solubility and resistant to biodegradation when precipitated from effluent and binds to soil limiting their bioavailability to microorganisms for biodegradation. Nonionic surfactants are favorable as hydrocarbon or oil solubilizer because they can perform at low temperatures, has low-foaming characteristics and relatively stable at high temperatures and under harsh chemical conditions. Therefore, there is a need for the usage of surfactant that is biodegradable and at the same time effective at solubilizing FFAs in POME before being released to streams. Thus, FFAs will be kept mobilized and readily available for biodegradation by microorganisms. Oleic acid is a long chain free fatty acid presents as the major fatty acid component $(40-52 \% \mathrm{w} / \mathrm{w})$ in palm oil. Oleic acid was selected for solubilization by biodegradable nonionic surfactant polyoxyethylene (100) stearate with the commercial name Myrj 59. The solubilizations were conducted with various concentrations of Myrj 59; below, at and above the critical micelle concentration (CMC). The amount solubilized was determined by gas chromatography (GC) using flame ionization detector (FID) technique. The solubilization process was confirmed by characterizing the solubilized oil using Fourier Transform infrared (FTIR) to observe changes in chemical bonds. Highest solubilization was achieved with Myrj 59 solution at above its CMC, solubilizing $516.31 \mathrm{ppm}$ oleic acid. The FTIR spectra showed strong peak at $2927 \mathrm{~cm}^{-1}$ with high intensity suggesting intermolecular hydrogen bonding between oleic acid and ethylene oxide (EO) groups of surfactant.
\end{abstract}

Keywords: Oleic acid, Myrj 59, critical micelle concentration, solubilization, GC-FID, FTIR

\section{INTRODUCTION}

About $0.5-0.75$ tones palm oil mill effluent (POME) is discharged for every one ton of palm oil fresh fruit bunch containing average of $25,000 \mathrm{mg} / \mathrm{L}$ biochemical oxygen demand (BOD), 55,250 $\mathrm{mg} / \mathrm{L}$ chemical oxygen demand, $19,610 \mathrm{mg} / \mathrm{L}$ suspended solids including high content of free fatty acids (FFAs) (Wong et al., 2009). POME is one of the sources of contamination in effluent that leads to problems such as clogging in drainpipes and sewer lines. In addition this oily wastewater could cause corrosion to the sewer pipes under anaerobic conditions (Lemus and Lau, 2002). Furthermore, oily effluent could also harm sensitive aquatic organisms and its ecosystems (Li et al. 2005).
Oleic acid is a long chain free fatty acid present in palm oil ( $\mathrm{Li}$ et al., 2005). It is a monounsaturated free fatty acid contributing $40-52 \%$ of total weight in palm oil. The molecular weight of oleic acid is $282.45 \mathrm{~g} \mathrm{~mol}^{-}$ ${ }^{1}$ with low solubility in water of $230.2 \mathrm{mg} / \mathrm{L}$ (Lim et al., 2005).

Surfactant, a term from combination of phrase 'surface active agent' is a substance with a chemical structure that preferably absorbs at surface as a result of the presence of both tail and head groups (Daintith, 1985). There are four categories of surfactants; anionic, cationic, nonionic and amphoteric. Anionic surfactants carry negative charge at the hydrophile head group while cationic surfactants carry positive charge at the

*Corresponding author: mpsatirawaty@frst.unimas.my 
hydrophile head group. Nonionic surfactants have no charge and normally have polyoxyethylenes polar groups that contribute to the water solubility of the surfactant. Amphoteric surfactants have both negative and positive charges at the hydrophile head groups (Myers, 2006). Surfactants are widely used in many applications including pharmaceuticals, biotechnology, oil recovery and also in our daily life needs like food and cosmetics.

Critical micelle concentration (CMC) is the concentration where the surfactant forms aggregates or micelles. Surfactant exists as free monomers in solution below this critical value. In a micelle structure the hydrophobic parts of the surfactant lies in the center of the aggregate while the hydrophilic parts is directed outward towards the aqueous medium. The critical concentration of micelles in aqueous solutions was believed to represents its' surface properties (Becher, 1959). CMC is used to characterize surfactants as this is the point where the physical properties such as surface tension, interfacial tension, conductivity, osmotic pressure, detergency, and emulsification change abruptly. Nonionic surfactant has very low CMC value compared to ionic surfactants (Rangel-Yagui et al., 2005).

Nonionic surfactants are good oil solubilizer compared to charged surfactants. This is because charged (ionic) surfactant will have repulsion interaction with oil (Peña and Miller, 2006) and would reduce the capability of surfactant to dissolve oil. In addition nonionic surfactants performed better in oil solubilization because they are more effective, less toxic, has lower aqueous solubility and are less affected by electrolyte concentration compared to ionic surfactants (Wrenn et al., 2009). In this study, the nonionic surfactant chosen is Myrj 59. This is a group of surfactant having straight chain of the hydrophobic tail. Surfactant with a straight chain of hydrophobic tail is better at solubilizing oil compared to branched chains (Myers, 2006).

Myrj 59 surfactant is a biodegradable nonionic surfactant of the polyoxyethylene sorbitan monofatty acids ester group. Polyoxyethylene (100) stearate or Myrj 59 has a chemical formula of $\mathrm{C}_{18} \mathrm{H}_{35} \mathrm{O}_{2}\left(\mathrm{C}_{2} \mathrm{H}_{4} \mathrm{O}\right)_{100} \mathrm{OH}$, with molecular weight of $4683 \mathrm{~g} \mathrm{~mol}^{-1}$ (Hait and
Moulik, 2001).

Lim et al. (2005) studied solubilization of three major free fatty acids (FFAs) of palm oil; palmitic, oleic and linoleic acids in ethoxylated surfactants; Tergitol T-S-5, Tergitol T-S-7, Neodol N-7, GE-460 and GE-690. The solubilization of the free fatty acids were found to be linear with surfactant concentration above CMC. The molar solubilization ratio (MSR) of palmitic acid decreases as the hydrophile lipophile balance (HLB) value of surfactant used increases which indicate that palmitic acid is hydrophobic. Maximum solubilization for oleic and linoleic acids were observed at higher surfactant HLB indicating that these acids are slightly hydrophilic compared to palmitic acid.

\section{MATERIALS \& METHODS}

\section{Materials}

Myrj 59 and oleic acid (90\% purity) are from Sigma Aldrich and were used as received without further treatment. Double distilled deionized water of at least $18 \mathrm{M} \Omega$ purified by Nano Ultra Pure Water System (Barnstead, USA) was used for making up solutions.

\section{Surfactant Critical Micelle Concentration (CMC)}

Critical micelle concentration (CMC) of Myrj 59 surfactant was determined by turbidimetry method. Turbidity measurements of surfactant solutions were carried out using $\mathrm{HACH}$ DR4000 (Vis) Spectrophotometer at $860 \mathrm{~nm}$ at room temperature. Double distilled deionized water was used as the reference solution. CMC of surfactant was the point where there is a sudden change in the plot of turbidity of surfactant against surfactant concentration. This is due to the presence of scattering surfactant species that are significantly greater in size than the monomeric solute (Myers, 2006).

\section{Oleic acid solubilization method}

Oil solubilization was conducted by formation of oil in water $(\mathrm{O} / \mathrm{W})$ emulsions containing fixed amount of surfactant solution and excess oleic acid. An amount of $10 \mathrm{ml}$ of surfactant below, at and above CMC was added to excess oleic acid in three $250 \mathrm{ml}$ Erlenmeyer flask. The mixture was then agitated on a rotary 
shaker $(150 \mathrm{rpm})$ at room temperature for a minimum of seven days to reach equilibrium solubilization (Li et al., 2005). After equilibrium solubilization was achieved, the emulsion was transferred to test tubes and left to stand for two days to separate unsolubilized oil phase from the aqueous phase (emulsion).

The concentration of solubilized oleic acid was measured by gas chromatography (GC) Hewlett Packard 6890 model using a flame ionization detector (FID) adopted from study by Diallo et al. (1994) and Dwarakanath and Pope (2000). One microlitre aliquot of a mixture of $1 \mathrm{ml}$ solution from the test tube (emulsion below the oil phase; pre-dried with water bath to eliminate water) and $1 \mathrm{ml}$ dichloromethane was injected into the GC column. The column was operated from 200 ${ }^{\circ} \mathrm{C}$ up to $265^{\circ} \mathrm{C}$ with the rate $10{ }^{\circ} \mathrm{C} / \mathrm{min}$ with helium as the carrier gas. The concentration of oleic acid solubilized in surfactant solutions is determined using calibrated plot of four different standard solutions of oleic acid; 200 ppm, 400 ppm, 600 ppm and 800 ppm.

\section{Surfactant-oil interaction}

Surfactant-oil interaction was studied using fourier transform infrared (FTIR) spectroscopy. FTIR analysis was carried out using Nicolet Avatar 370 DTGS spectrometer to observe the changes of bonds in the samples before and after solubilization. The emulsion samples (1 $\mathrm{ml}$ of aqueous phase from test tube pre-dried with water bath to eliminate water) were extracted with chloroform and infrared (IR) spectra were recorded as thin film (sodium chloride disc) for liquid sample.

The FTIR spectra were recorded in the range of $4000-500 \mathrm{~cm}^{-1}$ in the transmittance mode. IR spectrum of each pure surfactant was also recorded in the same range. The samples were mixed with Nujol solution and also recorded as thin film (sodium chloride disc) for liquid sample. IR spectrum of pure oleic acid was directly recorded as thin film (sodium chloride disc) as it is in liquid form.

\section{RESULTS \& DISCUSSION}

\section{Surfactant Critical Micelle Concentration (CMC)}

The CMC value of Myrj 59 determined by turbidimetry method is $1.4 \times 10^{-4} \mathrm{M}$. According to Drummond et al. (2004), the CMC value of Myrj 59 was only reported as lower than $10^{-5}$ M. The difference is attributed to different methods used to determine the CMC (Xu et al., 2008). There are many methods that had been used to determine CMC of surfactant depending on the availability, easiness of use, type of surfactant and its spectroscopic properties (Saux et al., 2004). CMC of surfactants are normally determined by surface tension measurement. Rajec and Paulenová (1994) used turbidimetry method to determine CMC of surfactant. However, CMC determination of the same surfactant by different experimental techniques including light scattering, conductivity and surface tension often would give different CMC values (Todorov et al., 2002). Hence, it is important to determine CMC of surfactant prior to next step of a study as different method could give different value of CMC for the same surfactant.

\section{Oleic acid solubilization}

Concentration of oleic acid solubilized in Myrj 59 solutions at concentration below CMC, at CMC and above CMC in average of three replicates are $39.25 \mathrm{ppm}, 70.15 \mathrm{ppm}$ and $516.31 \mathrm{ppm}$, respectively (Figure 1). It was evident that oleic acid solubilization by Myrj 59 solutions increase as the concentrations of surfactant solutions increase. Degree of solubilization depends on the concentration of surfactant used. The solubilization of oleic acid is greatly achieved by large micellar structures, which is well above CMC (10-100 times) of surfactant concentration (Myers, 2006). In addition, micelle volume also increases as the concentration increases, providing greater volume for oleic acid to partitionate into the micelle (Edwards et al., 1991). This is shown in the significant increase of oleic acid solubilization by Myrj 59 solutions from below CMC to above CMC. 


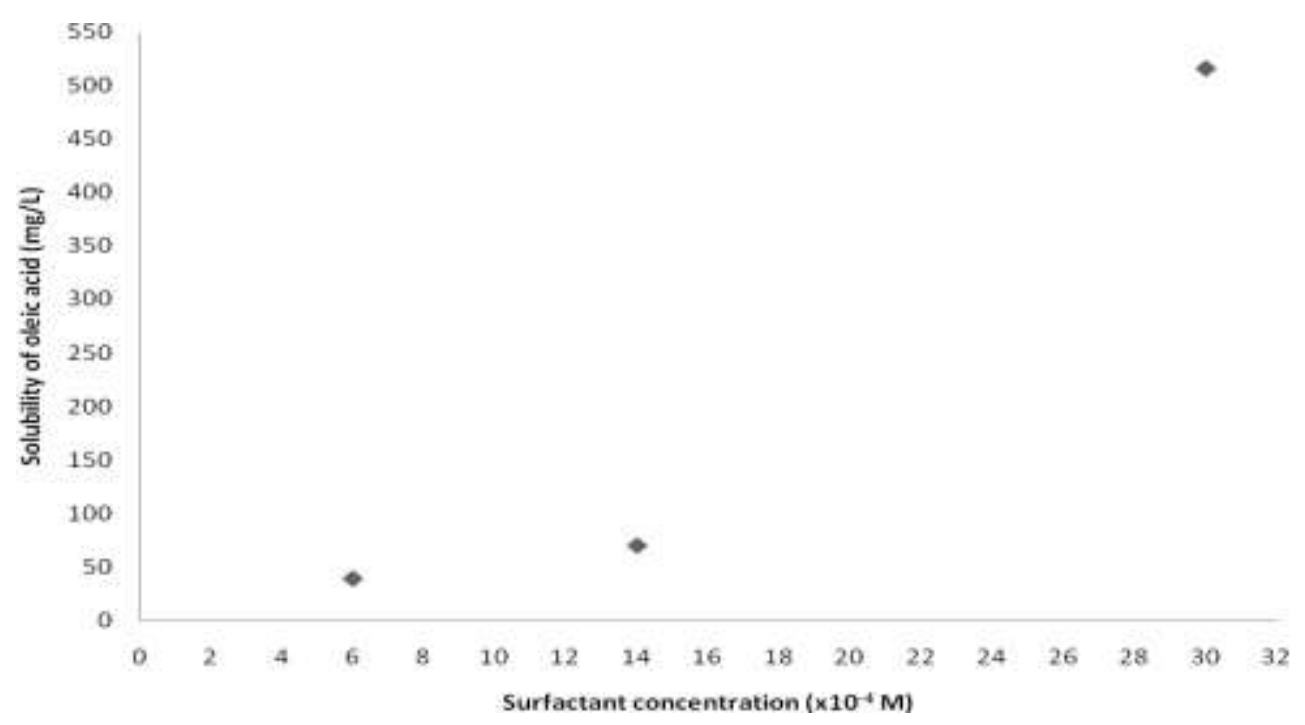

Figure 1. Solubilization of oleic acid by Myrj 59

The hydrocarbon tail of a surfactant molecule dissolves in hydrocarbon and non-polar solvents while hydrophilic head part dissolves in polar solvents especially water. Although oleic acid is hydrophobic, the slightly polar characteristic of oleic acid resulted in solubilization by Myrj 59 not only in the hydrophobic micellar core but also in the hydrophilic mantle (external) of micelles (Lim et al., 2005).

The nature of hydrophilic group in the structure of surfactant also contributed to the solubilization efficiency. The long hydrophilic group, polyoxyethylene (POE) chain of Myrj 59 resulting in solubilization of high amount of oleic acid. Long POE chain contribute to the dominant and strong hydrophilic interaction such as hydrogen bonding, dipole-dipole and dipole induced attraction forces with oleic acid that suppress the presence of hydrophobic Van der Waals forces (Xiarchos and Doulia, 2006). In addition, the low lipophilicity value of Myrj $59,3.55$ resulting in better interaction with slightly polar oleic acid (Cserháti and Forgács, 1997). Thus, oleic acid solubilized in the outer layer of micelles with its polar group $(\mathrm{C}=\mathrm{O})$ oriented towards the POE chains and its alkyl portion towards the micelle interior (Xiarchos and Doulia, 2006). Study by Lim et al., (2005) reported that oleic acid is favorable to be solubilized in the shallow palisade of the micelle of surfactants as well as in the core of the micelles or in the deeper palisade.

\section{Surfactant-oil interaction}

From IR spectrum of oleic acid (Figure 2b), several peaks were observed. $\mathrm{O}-\mathrm{H}$ stretch is very broad, starting at about $3300 \mathrm{~cm}^{-1}$ and then slopes into the $\mathrm{C}-\mathrm{H}$ absorption band. $\mathrm{C}-\mathrm{H}$ stretching band in $\mathrm{C}=\mathrm{C}-\mathrm{H}$ is observed at 3004 $\mathrm{cm}^{-1}$, asymmetric $\mathrm{CH}_{2}$ is at $2925 \mathrm{~cm}^{-1}$ while symmetric $\mathrm{CH}_{2}$ is at $2854 \mathrm{~cm}^{-1}$. The intense peak at $1711 \mathrm{~cm}^{-1}$ is $\mathrm{C}=\mathrm{O}$ stretch of the carboxyl group while at $1284 \mathrm{~cm}^{-1}$ exhibited the presence of $\mathrm{C}-\mathrm{O}$ stretching. Absorption band at $1464 \mathrm{~cm}^{-1}$ is attributed to $\mathrm{O}-\mathrm{H}$ in plane whereas the band at $938 \mathrm{~cm}^{-1}$ is attributed to O$\mathrm{H}$ out of plane. The bands at $723 \mathrm{~cm}^{-1}$ and $1412 \mathrm{~cm}^{-1}$ are assigned to $\mathrm{CH}_{2}$ rocking of methylenes longer than four carbon atoms and $\mathrm{CH}_{3}$ umbrella mode respectively. All the absorption bands observed are in agreement with oleic acid spectrum reported by $\mathrm{Wu}$ et al. (2004).

Meanwhile, for IR spectrum of Myrj 59 (Figure 2a), the broad band around $3440 \mathrm{~cm}^{-1}$ is due to $\mathrm{O}-\mathrm{H}$ stretch while absorption bands at $2922 \mathrm{~cm}^{-1}$ and $2852 \mathrm{~cm}^{-1}$ are attributed to $\mathrm{CH}_{2}$ asymmetric and $\mathrm{CH}_{2}$ symmetric stretch respectively. Bands at $1147 \mathrm{~cm}^{-1}, 1060 \mathrm{~cm}^{-1}$ and $964 \mathrm{~cm}^{-1}$ are assigned to $\mathrm{C}-\mathrm{O}-\mathrm{C}$ stretch. $\mathrm{CH}$ bending is observed at $1463 \mathrm{~cm}^{-1}$ and the bands at $1376 \mathrm{~cm}^{-1}$ and $1344 \mathrm{~cm}^{-1}$ are due to $\mathrm{CH}$ deformation of the methyl group (Kriegel et al., 2009). Absorption band at around 1742-1713 $\mathrm{cm}^{-1}$ is assigned to $\mathrm{C}=\mathrm{O}$ stretch. 
After absorption of oleic acid by surfactant solutions (Figure 2c), the $\mathrm{CH}_{2}$ asymmetric and $\mathrm{CH}_{2}$ symmetric stretch in the oleic acid shifted to 2927 and $2856 \mathrm{~cm}^{-1}$ respectively. From the spectrum in Figure 2c, it can be observed that the ethylene oxide (EO) chain of surfactants is linked with oleic acid through ester bonds at 1712-1709 $\mathrm{cm}^{-1}$ (Shinoda et al., 1996). The FTIR spectrum also showed strong peak at
$2956 \mathrm{~cm}^{-1}$ with high intensity, suggesting combination between oleic acid and hydrophobic groups of surfactants (Ibrahim et al., 2009). The carboxyl in oleic acid interacted with the EO chain of surfactant by intermolecular hydrogen bonding and this is observed at sharp intense band at $2927 \mathrm{~cm}^{-1}$ (Rangel-Yagui et al., 2005).

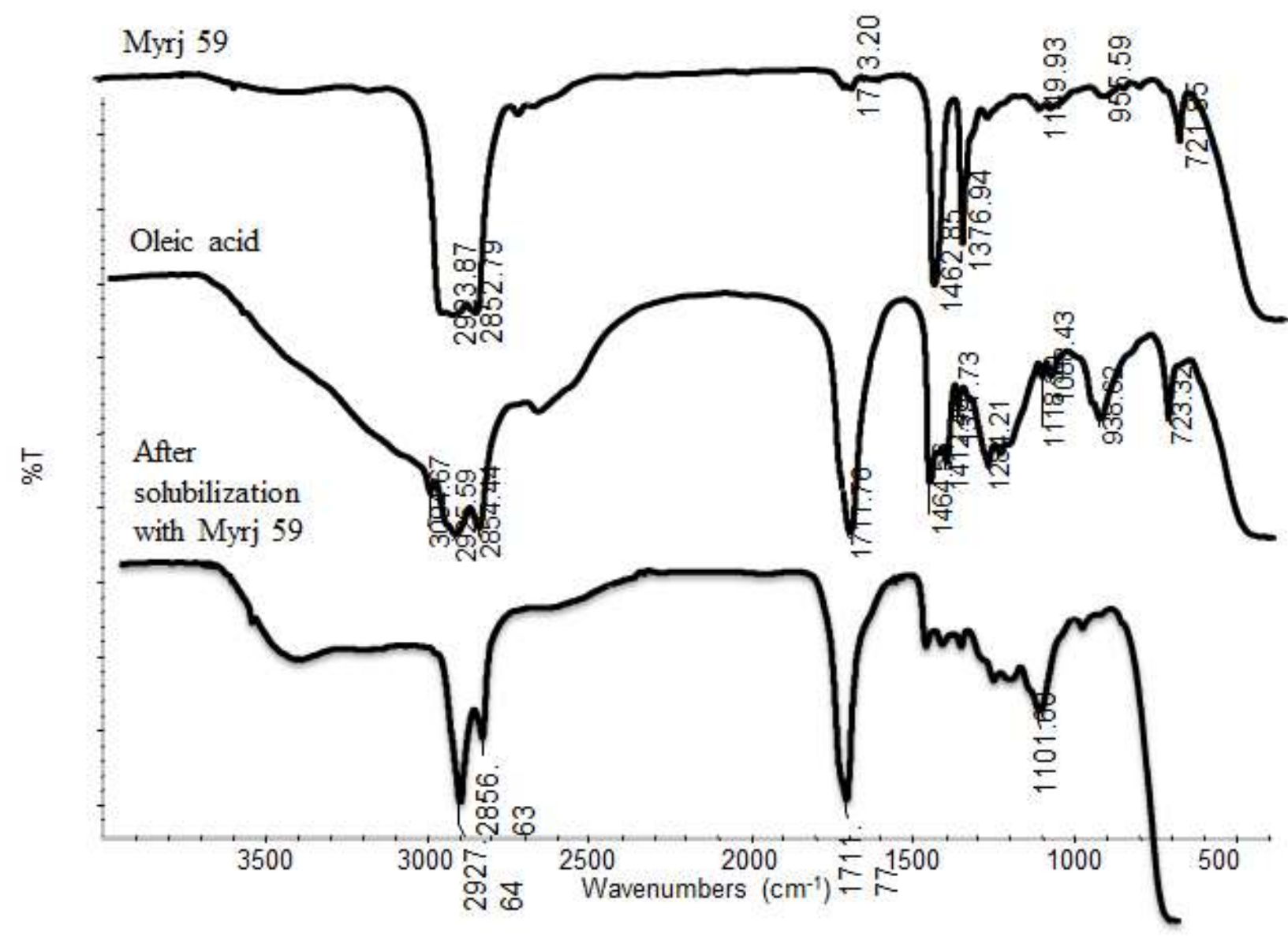

Figure 2. FTIR spectra of a) Myrj 59 b) Oleic acid and c) Solubilized oil by Myrj 59

\section{CONCLUSION}

The amounts of oleic acid solubilized by Myrj 59 solutions at below $\mathrm{CMC}$, at $\mathrm{CMC}$ and at above CMC are $39.25 \mathrm{ppm}, 70.15 \mathrm{ppm}$ and $516.31 \mathrm{ppm}$ respectively. Solubilization of oleic acid is highest at concentration of Myrj 59 surfactant above its CMC with long length of hydrophobic tail chain and hydrophilic head of EO chain that provide both hydrophobic and lipophilic character. Thus this would be promising to be applied to POME.
However, effect of temperature on the dissolution of oleic acid by nonionic surfactants must also be taken into account as POME is generated at high temperature of 80$90^{\circ} \mathrm{C}$ before discharged to environment. Benchmark studies must also be conducted to indentify optimum surfactant concentration on the removal of residual oil in POME. 


\section{ACKNOWLEDGEMENTS}

Thanks were extended to Universiti Teknologi Malaysia for providing the necessary facilities and also to the members of Physical Chemistry 2 laboratory, Universiti Teknologi Malaysia for help and research supports.

\section{REFERENCES}

Becher, P. (1959). Non-Ionic Surface-Active Compounds. I. Critical Micelle Concentrations of Water-Soluble EtherAlcohols. Journal of Physical Chemistry, 63: $1675-1676$.

Cserháti, T and Forgács, E. (1997). Separation and Quantitative Determination of NonIonic Surfactants Used as Pesticides Additives. Journal of Chromatography A, 774: 265-279.

Daintith, J. ed. (1985). Oxford Dictionary of Chemistry. New York: Oxford University Press.

Diallo, M. S., Abriola, L. M. and Weber, W. J. Jr. (1994). Solubilization of Nonaqueous Phase Liquid Hydrocarbons in Micellar in Palm Oil by Biodegaradable Ethoxylated Surfactants. Journal of Agricultural and Food Chemistry, 53 (11): 4476-4483.

Drummond, C., In, M. and Richetti, P. (2004). Behavior of Adhesive Boundary Lubricated Surfaces Under Shear: Effect of Grafted Diblock Copolymers. The European Physical Journal E, 15; 159-165.

Dwarakanath, V. and Pope, G. A. (2000). Surfactant Phase Behavior with Field Degreasing Solvent. Environmental Science and Technology, 34 (22): 4842-4848.

Edwards, D. A., Luthy, R. G. and Liu, Z. (1991). Solubilization of Polycyclic Aromatic Hydrocarbons in Micellar Nonionic Surfactant Solutions. Environmental Science \& Technology, 25 (1): 127-133.

Hait, S. K. and Moulik, S. P. (2001). Determination of Critical Micelle Concentration (CMC) of Nonionic Surfactants by Donor-Acceptor Interaction with Iodine and Correlation of CMC with Hydrophile-Lipophile Balance and Other
Parameters of the Surfactants. Journal of Surfactants and Detergents. 4 (3), 303-309.

Ibrahim, S., Ang, H. M. and Wang, S. (2009). Removal of Emulsified Food and Mineral Oils from Wastewater Using Surfactant Modofied Barley Straw. Bioresource Technology, 100: 5744-5749.

Kriegel, C., Kit, K. M., McClements, D. J. and Weiss, J. (2009). Electrospinning of Chitosan-poly(ethylene oxide) Blend Nanofibers in the Presence of Micellar Surfactant Solutions. Polymer, 50: 189-200.

Lemus, G. R. and Lau, A. K. (2002). Biodegradation of Lipidic Compounds in Synthetic Food Wastes During Composting. Canadian Biosystem Engineerings. 44: 6.33-6.39.

Li, Z., Wrenn, B. A. and Venosa, A. D. (2005). Anaerobic Biodegradation of Vegetable Oil and Its Metabolic Intermediates in OilEnriched Freshwater Sediments. Biodegradation, 16: 341-352.

Lim, T. Y., Li, J. L. and Chien, B. H. (2005). Solubilization of Selected Free Fatty Acids in Palm Oil by Biodegaradable Ethoxylated Surfactants. Journal of Agricultural and Food Chemistry, 53 (11): 4476-4483.

Myers, D. (2006). Surfactant Science. Third Edition. Hoboken: John Wiley \& Sons, Inc. pp 31, 198, 117-118.

Peña, A. A. and Miller, C. A. (2006). Solubilization Rates of Oils in Surfactant Solutions and Their Relationship To Mass Transport in Emulsions. Advances in Colloid and Interface Science, 123-126: 241-257.

Rajec, P and Paulenová, A. (1994). Micellar Enhanced Microfiltration of Strontium. Journal of Radioanalytical and Nuclear Chemistry, 183 (1): 109-113.

Rangel-Yagui, C. O., Hsu, H. W. L., Pessoa-Jr, A. and Tavares, L. C. (2005). Micellar Solubilization of Ibuprofen-Influence of Surfactant Head Groups on the Extent of Solubilization. Brazilian Journal of Pharmaceutical Sciences, 41 (2): 237-246. 
Shinoda, K., Carlsson, A. and Lindman, B. (1996). On the Importance of Hydroxyl Groups in the Polar Head-Group of Nonionic Surfactants and Membrane Lipids. Advances in Colloid and Interface Science, 64: 253-271.

Saux, T. L., Varenne, A. and Gareil, P. (2004). Determination of Aggregation Thresholds of UV Absorbing Anionic Surfactants by Frontal Analysis Continuous Capillary Electrophoresis. Journal of Chromatography A, 1038: 275-282.

Todorov, P. D., Kralchevsky, P. A., Denkov, N. D., Broze, G. and Mehreteab, A. (2002). Kinetics of Solubilization of n-Decane and Benzene by Micellar Solutions of Sodium Dodecyl Sulfate. Journal of Colloid and Interface Science, 245: 371-382.

Wong, Y. S., Kadir, M. O. A. B. and Teng, T. T. (2009). Biological Kinetics Evaluation of Anaerobic Stabilization Pond Treatment of Palm Oil Mill Effluent. Bioresource Technology, 100: 4969-4975.
Wrenn, B. A., Virkus, A., Mukherjee, B. and Venosa, A. D. (2009). Dispersibility of Crude Oil in Fresh Water. Environmental Pollution, 157 (6): 1807-1814.

Wu, N., Fu, L., Su, M., Aslam, M., Wong, K. C. and Dravid, V. P. (2004). Interaction of Fatty Acid Monolayers with Cobalt Nanoparticles. Nano Letters, 4 (2): 383-386.

Xiarchos, I. and Doulia, D. (2006). Effect of Nonionic Surfactants on the Solubilization of Alachlor. Journal of Hazardous Materials, 136 (3): 882-888.

Xu, Q., Nakajima, M., Ichikawa, S., Nakamura, N., Roy, P., Okadome, H. and Shiina, T. (2008). Effects of surfactant and electrolyte Concentrations on Bubble Formation and Stabilization. Journal of Colloids and Interface Science, 332 (1): 208-214. 
